\title{
Ensino/Aprendizagem da estatística promovendo o pensamento crítico utilizando o fórum de uma LMS
}

\author{
Rui João Teles Silva-Ramalho, Xosé Manuel Cid-Fernández, \\ ESE Paula Frassinetti, Portugal, Universidad de Vigo - Campus Ourense, España
}

\begin{abstract}
Resumen
Tendo por referência as diretrizes para o ensino superior que apontam para a centralidade do estudante no processo autónomo de aprendizagem ao longo da vida, implementamos uma metodologia de b-learning no ensino/aprendizagem da estatística, alicerçada na plataforma moodle usando a atividade "fórum" para potenciar o pensamento crítico. Os posts lançados a discussão, eram notícias dos meios de comunicação com um pequeno comentário, de forma a fomentar o pensamento crítico face ao modo como a informação é apresentada, tendo como objetivo desenvolver skills interpretativos e a literacia estatística
\end{abstract}

Palabras clave: estatística, pensamento crítico ,interação online, fórum

\section{Introdução}

O ensino da estatística hoje em dia, deve procurar novos métodos de ensino de forma a dar resposta ao novo paradigma de aprendizagem em que define o estudante como o agente central e ativo no processo de aprendizagem de forma a desenvolver competências de forma contínua ao longo da vida.

Neste contexto, as aprendizagens podem ser adquiridas colaborativamente, e neste sentido um ambiente virtual de aprendizagem constitui " um espaço fecundo de significação onde seres humanos e objetos técnicos interagem, potencializando assim a construção de conhecimentos, logo, aprendizagem"(Santos,2003, p.223).

Neste artigo, estudamos as interações realizadas no fórum reflexivo da unidade curricular de "Introdução à Estatística" na modalidade de b-learning, que tinha como objetivo, desenvolver skills interpretativos e a literacia estatística.

Em primeiro lugar procedeu-se à contextualização teórica do estudo, tendo sido apresentado o Modelo Community of Inquiry. De seguida, caracterizou-se o fórum reflexivo da unidade de introdução à estatística.Relativamente à metodologia, foi efetuada uma análise sociométrica através do software UNICET/NetDraw com o objetivo de estudar em detalhe os indicadores da rede. No final do estudo foi realizada uma interpretação das interações feitas pelos estudantes tendo em conta os contributos do modelo teórico apresentado.

\section{Contextualização teórica do estudo}

$\mathrm{O}$ modelo Community of inquiry ( $\mathrm{CoI}$ ) fornece um quadro teórico que apoia a investigação sobre a aprendizagem online. A aprendizagem colaborativa, tendo como suporte uma plataforma de e-learning tem sido estudada por vários investigadores dos quais destaco, Moore, Garrison e Bloom.

O modelo de CoI desenvolvido por Garrison, Anderson e Archer (2000) explora as relações entre os diversos intervenientes no processo educacional.Este modelo é composto por três elementos:- Presença Social;Presença cognitiva;- Presença de Ensino.Cada elemento tem definidas categorias e indicadores. Este modelo corrobora as teorias construtivistas da aprendizagem no ensino superior.

Garrison, Cleveland-Innes, e Fung(2004), Arbaugh e Hwang(2006) confirmam a estrutura do modelo de comunidade de inquirição, através de análises fatoriais.

\section{Presença social}

A presença social na aprendizagem online é a capacidade de os alunos interagirem uns com os outros e professor, através de uma comunicação mediada por instrumentos tecnológicos. Os alunos devem reconhecer que não estão ali por motivos puramente sociais, mas sim, envolvidos em objectivos comuns de investigação. A presença social, por si só, não garante o desenvolvimento do espírito crítico na aprendizagem. $\mathrm{O}$ aumento da sociabilidade dos participantes em cursos leva a uma maior interação,ajudando o desenvolvimento da presença cognitiva.

\section{Presença de Ensino}

A presença de ensino consiste no design, facilitação e dinamização dos processos cognitivos e sociais, com o objetivo de tranformar o conteúdo em resultados de aprendizagem e com significado educacional. A presença de ensino tem três componentes: 1- desenho intrucional; 2- facilitação do discurso; 3 - instrução direta;

O desenho instrucional caracteriza a estrutura do curso, descreve o processo com atividades, recorrendo a recursos em diferentes formatos, tais como, áudio, video e documentos que funcionam como suporte à realização das atividades. Para além disso, define também os aspetos da avaliação.

A facilitação do discurso pretende analisar a participação dos estudantes, em diferentes níveis de interação, intervindo o professor de forma a permitir tranformar a informação em conhecimento por cada um dos participantes. A instrução direta está muito ligada à facilitação do discurso o que faz com que alguns autores com por exemplo, Shea (2006) chame também a 
facilitação direta que resulta da junção entre instrução direta e facilitação,no entanto, estas divergências tem haver com a visão dos alunos, isto é, nem sempre distinguem se o professor esta a guiar a discussão ou a inquirir fazendo com que os estudantes tomem consciência do processo de inquirição.

\section{Presença Cognitiva}

A presença cognitiva é manifestada através da compreensão e construção de um significado promovendo a reflexão e um discurso estruturado. Esta presença segundo Garrison, Anderson e Archer é considerada uma característica que se evidência no ensino superior, sendo operacionalizada num modelo prático de inquirição, num processo de quatro fases: (1) uma tarefa desencadeadora; (2) exploração; (3) integração; (4) a resolução. Definimos a presença cognitiva como um ciclo de inquirição prática, onde os participantes se manifestam de forma deliberada, da compreensão do problema ou questão até à sua exploração, integração e aplicação. As duas primeiras fases são fácilmente atingivéis mas as duas últimas depende muito do papel do professor e do tempo fornecido para reflexão.

$\mathrm{O}$ pensamento crítico e de inquirição são processos reflexivos internos da mente de cada estudante, assumindo assim o modelo, uma sinergia entre a reflexão e a ação comunicativa.

\section{Dinâmica da sala de aula virtual - Fórum}

As tecnologias da informação vieram potenciar a comunicação entre o professor e os estundantes e entre os estudantes para além do espaço de sala de aula promovendo uma interação mais consistente: "interação na perspectiva de comunicação com o objetivo de influênciar o pensamento cítico e reflexivo"(Garrison,Cleveland-Innes,2005).

Os fóruns vieram introduzir uma nova forma de dinamizar a comunicação assíncrona entre o professor e os estudantes possibilitando a criação de vínculos que se manifestam de diferentes formas para além de uma simples troca de informação permitindo criar uma comunidade de aprendizagem, para além disso, perceber a presença de outros estudantes que não entrevêem nas aulas presenciais.Este meio de comunicação permite acesso diversificado a outras fontes de informação aleado a um tempo mais prolongado permitindo uma maior reflexão crítica do que nas aulas presenciais.Esta metodologia permite ter uma visão diferente sobre os conhecimentos dos estudantes promovendo a inclusão daqueles que não se mostram nas aulas presenciais.Os fóruns que conseguem captar o interesse do estudante , cria vínculos que ultrapassam a simples troca de informações entre estudantes, proporcionando a criação de uma comunidade virtual.Salmon(2000) posiciona a avaliação dos fóruns no papel do mediador e da mediação online, na análise de conteúdo das mensagens e no número de mensagens trocadas entre os estudantes e o professor.Os fóruns possibilitam uma extensão do espaço de sala de aula facilitando a aprendizagem e um crescimento da interação entre os estudantes e o estudante e professor de uma perspectiva de colaboração aumentando o sentimento de pertença à comunidade. Downes(2004) caracteriza uma comunidade de aprendizagem do seguinte modo:

- as tarefas desenvolvidas na comunidade reduzem o trabalho indívidual porque permite que os estudantes se ajudem mutuamente;- uma comunidade de aprendizagem poderá mostrar diferentes perspectivas sobre um conteúdo promovendo uma aprendizagem para além do conteúdo;

- a comunidade gera compromissos que não são possíveis de atingir quando um dos estudantes trabalha sózinho com o conteúdo;- a comunidade permite um apoio à aprendizagem através da colaboração, discussão e leitura de novas ideias e perspectivas de outros estudantes.

Todo este processo de interação entre estudantes e professor podem ser caracterizados a nível da presença cognitiva,social evidenciando o tipo de presença de ensino.

\section{Caracterização do Fórum}

O fórum em análise está inserido na unidade curricular de Introdução à Estatística do $3^{\circ}$ ano da licenciatura em Educação Básica de uma instituição do ensino superior particular na cidade do porto. A unidade curricular foi dinamizada na modalidade de b-learning. A avaliação é composta por três componentes de avaliação, fórum $(30 \%)$, teste escrito( $40 \%$ ) e projeto(30\%). O fórum foi dinamizado durante o meses de de março e abril, e teve como suporte à aprendizagem a LMS (Learning Management System) Moodle, um sistema de Gestão de cursos on-line. O fórum era do tipo standard de uso geral, isto é, um fórum aberto onde cada um pode começar um novo tema ou responder a algum já existente, priveligiando a comunicação assíncrona. Os temas a abordar teriam que ser noticias dos meios de comunicação, como jornais, revistas ou noticias da TV onde estivesse presente números ou conteúdos estatísticos. O âmbito educativo do fórum sendo um espaço de comunicação assíncrona torna-se fácil ir para além da discussão de conteúdos, incorporando atividades que promovam a confrontação e argumentação de diferentes opiniões. Esta perspectiva permite introduzir a metodología de análise de redes sociais de forma a caracterizar as interações que ocorram no fórum de discussão.

\section{Metodologia}

Trata-se de um estudo que analisa as interações do fórum de aprendizagem da unidade de introdução à estatística da licenciatura em educação básica.

O grupo de participantes são os 74 alunos inscritos na unidade que estão divididos em duas turmas em que apenas dois estudantes são do sexo masculino.

\section{Instrumentos}

A estrutura da rede vai ser analisada em função de varios indicadores e para o efeito utilizamos o software UNICET/NetDraw para explorar os indicadores de centralidade que nos permite conhecer as interações entre qualquer indivíduo ou indivíduos, partido essencialmente de dados qualitativos. 
Assim, as redes ou grafos criados constituem uma ferramenta importante para representar as interações entre indivíduos ou grupo de indíviduos. Nem sempre a representação gráfica é suficiente para caracterizar a rede em pormenor, por isso, vários investigadores têm desenvolvido instrumentos matemáticos específicos para a análise das redes de interação capazes de explicar a estrutura da rede, tanto no seu conjunto como individualmente. Os mais utilizados são os indicadores de centralidade que permitem analisar a rede quanto ao grau de conectividade.

O uso destas ferramentas computacionais, analíticas e gráficas permitem uma análise das interações entre os individuos de forma a interpretar a importância de cada membro dentro do contexto de análise.

Os elementos da rede são:-Nós -estudantes que se agrupam com o objetivo de trabalhar um tema em comum. A soma de todos os nós representa o tamanho da rede;-Vínculo -laços comunicacionais criados entre dois nós representados por linhas;-Fluxo - indica a direção e sentido do vínculo de um nó com outro representado através de uma linha com seta (sentido).Estes fluxos podem ser unidirecionais ou bidirecionais dependendo da comunicação estabelecida entre os nós.No caso de nós, que não estabeleçam qualquer tipo de interação com outro nó, isto é, ausência de vínculos, dizemos que o nó está isolado ou solto dentro da rede.

Os indicadores de rede a explorar nesta investigação são os seguintes:

Densidade - A densidade de uma rede mostra o valor em percentagem do grau de conectividade entre os estudantes. O cálculo faz-se pelo quociente entre o número de relações existentes com as relações possíveis multiplicando por cem. ( $\mathrm{D}=(\mathrm{RE} / \mathrm{RP}) \times 100)$

Inclusividade - Trata-se da percentagem de estudantes que participam nas interações da rede.

Centralidade - $\mathrm{O}$ grau de centralidade consiste no número de estudantes com os quais o nó está diretamente ligado.Onde também podemos observar o grau de entradas e saídas, isto é, a soma das interações recebidas pelo estudante e a soma das interações dinamizadas pelo estudante respectivamente.Nas tabelas de output também aparece o grau de saída e entrada normalizado que representam as percentagens dos referidos graus.

Centralização - o grau de centralização permite ver se existem estudantes claramente centrais na dinâmica de interação, ou seja, os que estão mais ligados.A existência de valores baixos indica que existem actores pouco ligados.

Intermediação - 0 grau de intermediação (Betweenness) permite analisar o número de vezes que que este aparece nos caminhos que ligam todos os pares de nós da rede, isto é, o número de pares de estudantes que o estudante é capaz de ligar.

Proximidade - o grau de proximidade (Closennesse) é a capacidade de um nó se ligar a todos os estudantes de uma rede. O cálculo é feito contando todas as distâncias geodésicas do estudante para se ligar aos restantes. Valores altos de proximidade indicam uma melhor capacidade dos estudantes se ligarem com os restantes estudantes da rede.

\section{Procedimentos}

Neste estudo, foi seleccionado o "fórum reflexivo" que corresponde à atividade de confronto e argumentação sobre noticias que envolvam informação sobre a qual seja possível comentar estatísticamente. Efectuamos uma análise de todas as intervenções dos estudantes de modo a quantificar e tipificar as relações existentes na rede de comunicação. A seguir, foi construída a matriz sociométrica a partir da qual foram calculados os indicadores da rede.Finalmente procedeu-se à interpretação dos dados recolhidos tendo por base o modelo teórico apresentado.

\section{Apresentação dos resultados}

$\mathrm{Na}$ figura 1, observamos os vínculos criados pelos estudantes que participaram no fórum.

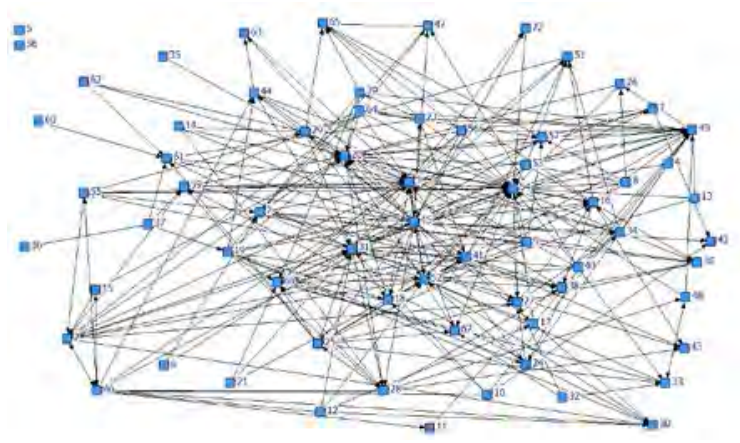

Figura 1.Rede de interações do fórum reflexivo

Identificamos na figura fluxos unidirecionais, ou seja, participantes que realizaram interações, mas não receberam interações e fluxos bidirecionais, ou seja, participantes que realizaram e receberam interações. Existem claramente sujeitos cujas as interações bidirecionais foram bastante elevadas e também observamos apenas dois nós isolados, isto é, não realizaram qualquer interação.

Apresentaremos agora os indicadores da análise sociométrica das interações:

- Densidade - O cálculo da densidade foi cálculado sem recurso ao software, através da seguinte fórmula: $\mathbf{D}=(\mathbf{R E} / \mathbf{R P}) \mathbf{x 1 0 0}$. A densidade desta rede é de $6,2 \%$ o que permite concluir que a conetividade da rede analisada é baixa pois das 2211 relações possíveis apenas se realizaram 306. - Inclusividade - Dos sessenta e sete participantes apenas 2 não fizeram nem receberam algum tipo de interação, o que dá uma inclusividade à rede de 97\%, isto é, observamos que o grupo não é segregativo.Centralidade - Analisando a tabela 1, verificamos que existe um estudante que se destaca a nível de entradas no fórum com 34 posts e outro estudante que se destaca com 24 comentários que recebeu de um post, mas em média existem 4.567 interações por participante. 
Tabela 1.

Resultados do grau de centralidade da rede dos participantes

\begin{tabular}{|c|c|c|c|c|}
\hline $\begin{array}{c}\text { Estatísticas } \\
\text { Descritivas }\end{array}$ & $\begin{array}{c}\text { Grau de } \\
\text { saída }\end{array}$ & $\begin{array}{c}\text { Grau } \\
\text { de } \\
\text { entrada }\end{array}$ & $\begin{array}{c}\text { Grau de saida } \\
\text { normalizado }\end{array}$ & $\begin{array}{c}\text { Grau de } \\
\text { entrada } \\
\text { normalizado }\end{array}$ \\
\hline Média & 4.567 & 4.567 & 6.920 & 6.920 \\
\hline $\begin{array}{c}\text { Desvio } \\
\text { padrão }\end{array}$ & 4.820 & 5.594 & 7.303 & 8.475 \\
\hline Soma & 306.00 & 306.00 & 463.636 & 463.636 \\
\hline Variância & 23.231 & 31.290 & 53.330 & 71.833 \\
\hline Mínimo & .000 & .000 & .000 & .000 \\
\hline Máximo & 34.000 & 24.000 & 51.515 & 36.364 \\
\hline
\end{tabular}

Centralização - Como os dados da tabela 2, afirmamos que estamos perante um grau moderado, o que significa que não existindo estudantes centrais existem alguns que se destacam na comunicação com os colegas.Por outro lado, o grau de entrada permeti aferir que a participação com comentários a posts foi menor que as propostas de temas por estudante.

Tabela 2.

Dados de centralização da rede

Centralização da rede (Grau de saída)

Centralização da rede (Grau de entrada)

$45,271 \%$

Intermediação -Na tabela 3, vemos nos dados uma percentagem de intermediação a variar entre 17.729 e zero o que em média dá $1,920 \%$

Tabela 3.

Estatísticas descritivas do grau de intermediação

\begin{tabular}{|c|c|c|}
\hline $\begin{array}{l}\text { Estaísticas } \\
\text { descritivas }\end{array}$ & $\begin{array}{l}\text { Grau de } \\
\text { intermediação }\end{array}$ & $\begin{array}{l}\text { Grau de } \\
\text { intermediação } \\
\text { normalizado }\end{array}$ \\
\hline Média & 82.388 & 1.920 \\
\hline Desvio Padrão & 172.921 & 4.031 \\
\hline Soma & 5520.000 & 128.671 \\
\hline Variância & 29901.670 & 16.247 \\
\hline Mínimo & .000 & .000 \\
\hline Máximo & 760.556 & 17.729 \\
\hline
\end{tabular}

Proximidade - Podemos verificar na tabela 4 que em média, cada estudante se consegue ligar A aproximadamente a 8 estudantes.

Tabela 4.

Estatísticas descritivas gerais do grau de proximidade.

\begin{tabular}{|l|l|}
\hline Estaísticas descritivas & Grau de proximidade \\
\hline Média & 8.360 \\
\hline Desvio Padrão & 3.903 \\
\hline Soma & 560.152 \\
\hline Variância & 15.233 \\
\hline Mínimo & 1.493 \\
\hline Máximo & 12.692 \\
\hline
\end{tabular}

De seguida vamos observar redes em que os termos estatísticos são utilizados nas interações entre os estudantes com a seguinte legenda para todas as figuras seguintes:- Quadrado Vermelho-"Participantes que mencionam "termo estatístico"; Quadrado Azul Participantes que não mencionaram "termo estatístico"; - Quadrado Amarelo -“Moderador”.

Podemos observar na figura 2, que em 67 estudantes apenas 9 usam o termo mediana nas suas interações

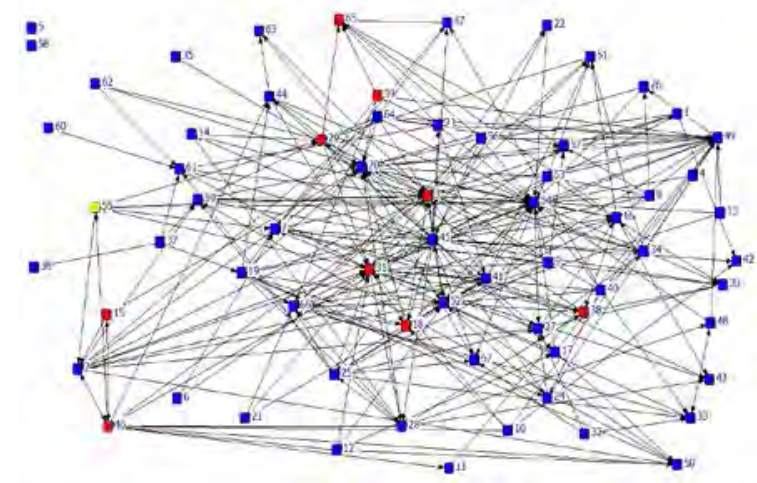

Figura 2. Grafo com os nós que mencionam "mediana"

$\mathrm{Na}$ figura 3 observamos que em 67 estudantes 56 utilizam a palavra média.

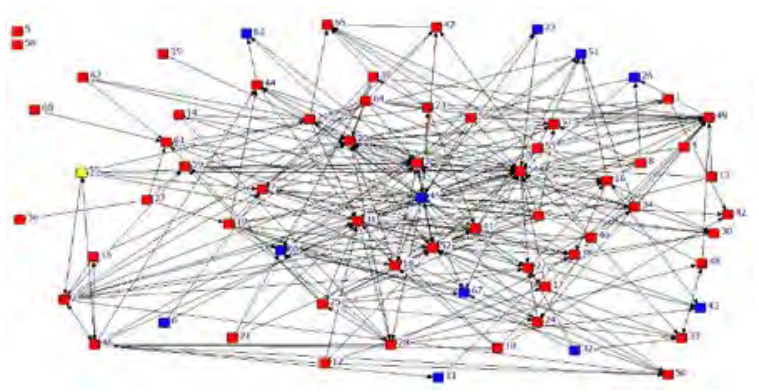

Figura 3. Grafo com os nós que mencionam " média"

$\mathrm{Na}$ figura 4 vemos que 67 estudantes dezassete aborda o conceito moda

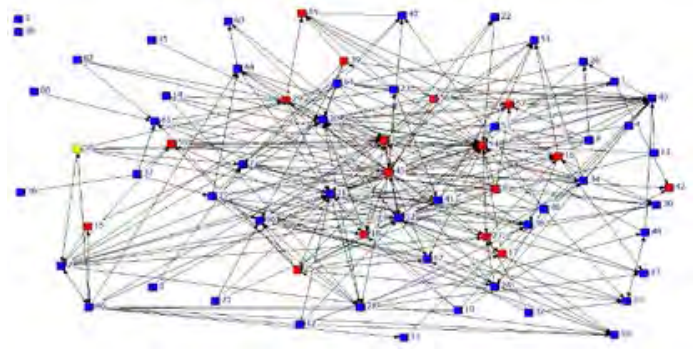

Figura 4: Grafo com os nós que mencionam "moda"

Na figura 5 vemos que em 67 estudantes apenas 2 falam do termo varância.

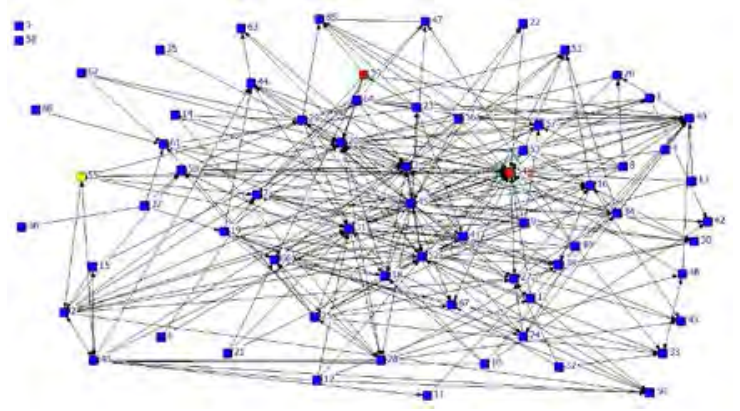

Figura 5: Grafo com os nós que mencionam "variância" Na figura 6 vemos que em 67 estudantes 5 mencionam o termo desvio-padrão. 


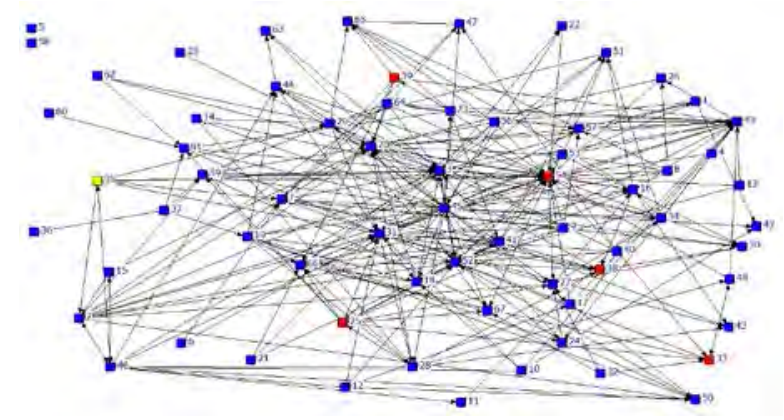

Figura 6: Grafo com os nós que mencionam "desvio padrão"

Apresentamos de seguida uma sistematização da análise do fórum tendo por base o modelo COI, evidenciando as três presenças, cognitiva, pedagógica e social.

Tabela 5.

Exemplos de interação cognitiva, pedagógica e social

\begin{tabular}{|l|l|}
\hline $\begin{array}{l}\text { Natureza } \\
\text { Interações }\end{array}$ & Exemplos \\
\hline Cognitiva & $\begin{array}{l}\text { "É certo como já foi referido nos } \\
\text { comentários anteriores que é o meio de } \\
\text { transporte mais seguro, no entanto é } \\
\text { sempre importante ter presente que } \\
\text { quando ocorre um acidente aéreo a } \\
\text { probabilidade de haver vítimas mortais } \\
\text { é muito superior ao acidente de viação. } \\
\text { Na minha opinião, acho é assustador a } \\
\text { percentagem de 67,57\% causada por } \\
\text { erros humanos." }\end{array}$ \\
\hline "Agradeço o vosso envolvimento e \\
participação, no entanto, solicito \\
intervenções com referência a \\
conteúdos abordados nas aulas, como \\
as medidas de localização e dispersão. \\
Esperando que este pedido seja \\
interiorizado... \\
Bons Post's"
\end{tabular}

\section{Conclusões}

As análises efectuadas permitem-nos afirmar que estamos numa rede de comunicação bastante inclusiva, pois apenas dois estudantes não estabeleceram qualquer tipo de interação.No entanto, a conetividade entre os estudantes é moderada porque a densidade da rede diz-nos que as interações realizadas foram em número menor que as possíveis.A centalidade da rede é moderada, verificando-se uma dispersão de comunicação pela maioria dos estudantes, o que faz com que não haja estudantes que se destacam na comunicação na rede.No entanto,observamos que o grau de saída é superior ao grau de entrada, isto significa, que os comentarios críticos às noticias foram feitos em menor número do que os temas propostos por cada um dos estudantes, e para além disso estes números permite-nos afirmar que não existiu nenhuma concentração especial em qualquer noticia. A intermediação que é considerado o indicador que permite fazer pontes entre outros estudantes foi um valor baixo, mas no geral a amplitude de valores foi um número razoável.Quanto à próximidade verificamos que em média um estudante consegue atingir cerca de oito estudantes num post, o que significa alguma dinâmica na discussão. Relativamente aos grafos apresentados com os termos estatísticos utilizados na discussão, verificamos que são utilizados mais os termos das medidas de localização do que as medidas de dispersão e o mais referido é o termo média. $\mathrm{Na}$ análise de conteúdo das participações no fórum, e tendo em conta o modelo de Garrison, pode concluir-se que existem interações das diversas naturezas: social, pedagógica e cognitiva, sendo esta a que tem maior representatividade, o que está de acordo com os objetivos do fórum.Uma abordagem futura desta metodogia de trabalho terá que ter uma maior intervenção por parte do professor de forma a incrementar a componente pedagógica para potênciar a parte cognitiva, com a utilização dos termos estatísticos nas análises críticas às noticias de forma a melhorar a literacia estatística de cada estudante.

\section{Referências}

Arbaugh, J. B., \& Hwang, A. (2006). Does "teaching presence" exist in online MBA courses? The Internet and Higher Education, 9(1), 9-21.

Downes, S. (2004). Learning in Communities. Recuperado em http://www.downes.ca/post/42101

Garrison, D., Anderson, T. \& Archer, W. (2000). Critical inquiry in a text-based environment: Computer conferencing in higher education. The internet and Higher Education, 2 (2-3),87-105. Recuperado de http://www.sciencedirect.com

Garrison D. R. \& Cleveland-Innes M.(2005). Facilitating cognitive presence in online learning: interaction is not enough. American Journal of Distance Education, n. 19, v.3, p.133-148

Garrison, D. R., Cleveland-Innes, M., \& Fung, T. (2004). Student role adjustment in online communities of inquiry: Model and instrument validation. Journal of Asynchronous Learning Networks, 8(2), 61-74. Recuperado de http://www.sloan-c.org/publications/jaln/v8n2/pdf/ v8n2 garrison.pdf

Gelman, A. \& Nolan, D. (2002). “Teaching Statistics: A bag of tricks". Oxford University,New York.

Scott, J. (2000). Social network analysis: a handbook (2nd). London: Sage Publications

Salmon, G. (2000). E-Moderating. The Key to Teaching and Learning Online.London:Kogan Page.

Santos, E. O. D. (2003). Articulação de saberes na EAD online. Por uma rede interdisciplinar e interativa de conhecimentos em ambientes virtuais de aprendizagem. In M. Silva (2003). Educação online (pp. 218-230). São Paulo: Edições Loyola 